\title{
Ureteral Stent Placement Following Ureteroscopy Increases Emergency Department Visits in a Statewide Surgical Collaborative
}

\author{
Spencer C Hiller ${ }^{1}$, Stephanie Daignault-Newton ${ }^{1}$, Hector Pimentel ${ }^{2}$, Sapan N Ambani ${ }^{1}$, \\ John Ludlow ${ }^{3}$, John M Hollingsworth ${ }^{1}$, Khurshid R Ghani ${ }^{1}$, Casey A Dauw ${ }^{1}$ \\ ${ }^{1}$ University of Michigan Department of Urology, Ann Arbor MI \\ ${ }^{2}$ Spectrum Health, Grand Rapids MI \\ ${ }^{3}$ Western Michigan Urological Associates, Holland MI
}

\section{Abstract}

Introduction: Ureteral stents are commonly placed after ureteroscopy (URS). Though studies indicate that stents are associated with patient discomfort, their impact on downstream health services use is unclear. We examined patterns of stent utilization in Michigan and their association with unplanned healthcare encounters.

Methods: We used the Michigan Urologic Surgery Improvement Collaborative's Reducing Operative Complications from Kidney Stones (MUSIC ROCKS) clinical registry to identify URS cases between 2016 and 2019. Factors associated with stent placement were examined using bivariate and multivariable statistics. Using multivariable logistic regression, we evaluated whether stent placement was associated with emergency department (ED) visits and hospitalizations within 30 days.

Results: We identified 9662 URS and a stent was placed in 7025 (73\%) of these. Frequency of stent use across the 137 urologists varied $(11-100 \%, \mathrm{p}<0.001)$, and was not associated with total case volume. Factors associated with stent use included age and stone size. Pre-stented cases and renal stones had a decreased odds of stent placement. On multivariable analysis after adjusting for risk factors, stent placement was associated with a 1.25 higher odds of ED visit (OR 1.25; 95\%CI $1.01-1.54 \mathrm{p}=0.043$ ) but not hospitalization (OR 1.28; 95\%CI 0.94-1.76 p=0.12). In a single high-volume practice, $0.5 \%$ of cases that omit a stent required urgent stenting postoperatively.

Conclusion: There is substantial variation in the use of stents in Michigan, irrespective of case volume. Stent placement significantly increased the odds of an ED visit after surgery. Importantly, stent omission rarely required subsequent urgent stent placement.

\section{Keywords}

Ureteral stents; ureteroscopy; urinary stone disease; emergency department

Corresponding Author: Spencer C Hiller, University of Michigan, Department of Urology, 1500 E Medical Center Dr, 3875 Taubman Center, Ann Arbor, MI 48109, Phone: 734-936-7030, Fax: 734-232-2400, spehill@ med.umich.edu. 


\section{Introduction:}

Ureteroscopy (URS) is the most frequently performed surgical procedure for urinary stone disease. ${ }^{1}$ In the United States, as many as $15 \%$ percent of patients undergoing URS have an emergency department (ED) visit or hospitalization postoperatively, amounting to a significant financial burden. ${ }^{2}$ Thus, efforts targeted toward reducing such unplanned visits would result in significant cost savings for the healthcare system. Pain and hematuria are among the most common chief complaints for these visits ${ }^{2,3}$, symptoms commonly attributed to a ureteral stent. ${ }^{4}$ However, the impact of stent placement on subsequent healthcare utilization remains unclear.

The American Urological Association and European Association of Urology's guidelines on the surgical management of urinary stones advocates for stent omission in the setting of uncomplicated URS. ${ }^{5} 6$ Despite these guidelines, stents are commonly placed, with large series demonstrating placement rates ranging from $66 \%$ to $84 \%,{ }^{3,7}$ Though a recent analysis from the Cochrane Database concluded that stent placement after URS may slightly reduce the number of unplanned healthcare visits following surgery, this finding was tempered by very low certainty of evidence. ${ }^{8}$

In this context, we used the Michigan Urological Surgery Improvement Collaborative's (MUSIC) Reducing Operative Complications from Kidney Stones (ROCKS) registry to characterize the patterns of stent use following URS in the state of Michigan. In particular, we define factors associated with ureteral stent utilization as well as determine the association between stent placement and unplanned healthcare encounters following URS. Our goal is to learn from these analyses to better inform patients undergoing URS of the risks and stimulate future research to develop interventions that reduce unnecessary ED visits and hospitalizations after surgery.

\section{Methods:}

Data source:

Established in 2011, MUSIC is a statewide quality improvement (QI) consortium formed in partnership with Blue Cross Blue Shield of Michigan. The ROCKS initiative was launched in 2016 with the overarching goal of reducing unplanned healthcare encounters following URS. This prospective clinical registry is comprised of a diverse collection of academic and community urology practices throughout the state. Currently, 37 practices participate in ROCKS, accounting for greater than $90 \%$ of the urologists within Michigan. Methods of data collection have been previously described. ${ }^{9,10}$ To ensure data quality, the coordinating center performs regular on-site data audits. Each practice has obtained an exemption or approval by the local institutional review board for participation in the collaborative.

\section{Study cohort:}

We included all primary URS within the registry occurring from June 2016 to May 2019. We excluded cases on patients $<18$ years old, synchronous bilateral surgery, and staged cases which we defined as 2 or more ipsilateral URS within a 4 week period. We chose to exclude staged cases as they are likely to be left with a stent to facilitate the secondary procedure. 
We additionally excluded procedures for stones $>20 \mathrm{~mm}$, as there are few within the registry $(\mathrm{n}=129)$ and are not likely to be considered for stent omission.

\section{Outcomes and statistical methods:}

We characterized cases with and without stent placement across a range of demographic and clinical measures. Demographic factors included age, gender, and insurance type. Clinical factors included body mass index (BMI), Charlson Comorbidity Index $(\mathrm{CCI})^{11}$, urine culture (positive, negative, not performed), presence of a stent prior to URS (pre-stented), stone size ( $5 \mathrm{~mm},>5 \mathrm{~mm}$ to $\unlhd 10 \mathrm{~mm},>10 \mathrm{~mm}$ ), stone location (renal, ureteral, both), ureteral access sheath (UAS) use, and occurrence of an intraoperative ureteral perforation or bleeding that precluded case completion. Categorical variables were compared using Chi-squared tests; continuous variables were compared using a t-test or Wilcoxon rank-sum. Provider- and practice-level variation in stent utilization across the ROCKS registry was described using proportions and differences tested using a Wald chi-square. Correlation between URS case volume and stent placement rate at a surgeon- and practice-level was calculated using Spearman correlation. The count and 95\% exact binomial confidence interval are reported. For reliability purposes, only providers or practices with $\geq 10$ URS in the registry were included in the provider and practice- specific analysis.

Since a potential ramification of stent omission is the need for urgent stent placement, we performed an independent chart review of all cases with stent omission with a 30-day ED visit at a single practice to understand the magnitude of this complication.

We used a multivariable logistic regression mixed model with provider nested in practice as random effects as well as BMI, CCI, urine culture result, pre-stent status, stone size, stone location, UAS use, and occurrence of an intraoperative ureteral perforation or bleeding that precluded case completion as fixed effects to determine the factors independently associated with stent placement. Two separate logistic regression mixed models were constructed to assess the association of stent placement with ED visit and with hospitalization within 30-days as dependent variables. These models also included provider nested in practice as random effects as well as BMI, CCI, urine culture result, pre-stent status, stone size, stone location, UAS use, and occurrence of an intraoperative ureteral perforation or bleeding that precluded case completion as fixed effects. To address the significant difference in rates of stent placement between pre-stented and non pre-stented cases, analysis for confounding was performed assessing the difference in odds of an ED visit between these two groups via the Breslow-Day test for homogeneity of odds ratios.

Propensity score analyses were performed as a sensitivity analysis to address potential confounding. In short, the propensity for stenting was estimated from the logistic model for stent placement described above. The propensity score distribution by stent placement status is presented in Figure 3. Greedy one-to-one matching was performed with an absolute difference of less than 0.001 between propensity scores of stented to non-stented patients, resulting in 1307 matched pairs. Odds ratios among the matched pairs is presented and McNemar's test used to assess significance. Additionally, inverse probability treatment weighting propensity score logistic models were performed with multiple propensity score 
trimming cut-offs based upon propensity score overlap to provide a range of effect size estimates for ranges of confounding adjustment.

The analysis was completed with 2-sided significance testing assuming a type I error of 0.05 using SAS 9.4 (SAS Institute Inc., Cary, NC).

\section{Results:}

We identified a total of 9662 URS performed between June 2016 to May 2019. Overall, a stent was placed in $72.7 \%$ of URS; $71.4 \%$ of cases for ureteral stones, $72.6 \%$ of cases for renal stones and $74.9 \%$ of cases when both a ureteral and renal stone were present.

Table 1 displays the demographic and clinical characteristics of cases in our analysis. There were significant differences between cases with and without a stent placed with regard to age, gender, insurance, pre-stenting, UAS use, intraoperative complication, stone size and location. Cases with stent placement tended to be larger and without pre-stent.

There were 137 surgeons from 24 practices who had performed at least 10 URS. We observed significant variation in the frequency of stent use at both a provider and practice level. The mean frequency of stent placement among urologists was $74.8 \%$ and varied from $10.7 \%$ to $100 \%$ ( $\mathrm{p}<0.001$; Figure 1). Eight urologists (6\%) placed a stent in all URS and 120 urologists $(88 \%)$ placed a stent in $250 \%$ of URS. Academic practices (associated with a residency program) had a significantly lower rate of stent placement compared to non-academic practices ( $67.9 \%$ vs $75.7 \%$ respectively, $\mathrm{p}<0.001)$. There was a broad range of stent usage irrespective of total URS volume (urologists with 250 URS highlighted in red, Figure 1). Within the 24 practices with $\geq 10$ URS, stent placement rates ranged from $33.6 \%$ to $100 \%$ ( $\mathrm{p}<0.001$; Figure 2, bars). Even within individual practices, the rates of stent placement varied between urologists irrespective of case volume (Figure 2, bubbles). We found no significant correlation between total URS case volume and stent placement rate amongst urologists as well as practices $(-0.1,95 \%$ CI $[-0.26-+0.07]$, p-value $=0.24$ and $-0.2595 \%$ CI [ $-0.58-+0.19]$, p-value $=0.27$ respectively $)$.

The results of our multivariable model, accounting for provider- and practice-level variation, indicated that several factors were independently associated with stent utilization (Table 2). In particular, age, pre-stenting, stone size, stone location and UAS use significantly impacted the odds of stent placement. Those with larger stones (>5mm- $\triangle 10 \mathrm{~mm}$ : OR 1.89 and $>10 \mathrm{~mm}$ : OR 4.68) had a significantly higher odds of stent placement. However, those with stones located within the kidney (OR 0.69) had decreased odds of stent placement relative to the ureter. Cases with a UAS had 5-fold higher odds of having a stent placed (OR 5.43). However, pre-stented cases had 75\% lower odds of having a stent placed (OR 0.25).

The overall rate of an ED visit within 30-days of URS was 8.1\% while the rate of hospitalization was 3.5\%. Reasons for ED visits are compared between those with and without a stent in Table 3. Stented and unstented patients had significantly different unadjusted rates of $\mathrm{ED}$ visits ( $8.5 \%$ vs $7.1 \%$ respectively; $\mathrm{p}=0.02)$ and hospitalization $(3.8 \%$ vs $2.9 \%$ respectively; $\mathrm{p}=0.03$ ) (Table 1 ). After controlling for patient, provider, and practicelevel differences on multivariable analysis, stent placement during URS was independently 
associated with a 1.25 higher odds of an ED visit within 30-days (OR 1.25, 95\% CI

$1.01-1.54 ; \mathrm{p}=0.043$ ) but not significantly associated with postoperative hospitalization (OR $1.28,95 \%$ CI $0.94-1.76 ; p=0.12$ ). These associations persisted on sensitivity analysis with propensity score models (Table 5). The odds of an ED visit was not significantly different between those with and without a pre-stent (OR 1.28, 95\% CI: $0.96-1.70$ vs OR 1.08 , $95 \%$ CI: $0.87-1.34$ respectively, $\mathrm{p}=0.36$ ). At a single practice, of the 399 cases without intraoperative stent placement, only $2(0.5 \%)$ required urgent stenting.

\section{Discussion:}

In our study, representing a diverse group of urology practices, we found that stents were placed in nearly three-quarters of URS. Patterns of use varied greatly between individual urologists and practices. Several factors were independently associated with stent placement including age, stone size, and UAS use while pre-stenting and stones located in the kidney were associated with a lower odds of stent placement. Stent placement had important implications for patients in that they had higher odds of an ED visit within 30 days of surgery. Additionally, stent omission had a low rate of subsequent urgent stent placement. Collectively, these findings suggest that the decision to place a stent is influenced by both patient- and surgeon-level factors but its use is not inconsequential.

There is currently insufficient evidence to accurately characterize every factor that contributes to the decision to place a stent. Though guidelines advocate for stent omission in uncomplicated URS, ${ }^{5}, 6$ the definition of uncomplicated URS remains ambiguous. Investigators representing the Clinical Research Office of the Endourological Society identified presence of an intraoperative complication, stone impaction, increasing operative time and stone burden and increased patient age as predictors for stent placement. ${ }^{7}$ These findings are consistent with the results of our study.

In a survey of Endourological Society members, $64 \%$ of respondents indicated that they utilize a stent in every URS. ${ }^{12}$ When limited to the United States, $76 \%$ and $81 \%$ of respondents indicated placing a stent in three-quarters of URS for ureteral and renal stones, respectively. ${ }^{13}$ In the present study, universal stent use was observed in only $6 \%$ of providers. Regardless, it appears clear that stents are placed frequently. Variation between prior studies and our own only further highlights the inconsistencies in practice patterns surrounding stent placement.

An important finding from our study relates to the implications of stent placement on patients and the healthcare system. Results of our logistic regression model indicate that stent placement is associated with a higher odds of ED visit following URS. These findings differ from a recent Cochrane review that included 16 randomized controlled trials and a total of 1970 patients. The authors concluded that placement of a stent may slightly reduce the risk of a ED visit but they acknowledge a high level of uncertainty of this finding and graded the quality of evidence as very low. ${ }^{8}$

Our findings must be viewed within the context of some limitations. Although the MUSIC registry includes a wide range of clinical and surgical variables, it is not exhaustive thus 
introducing the possibility of confounding. Specifically, the registry does not include the size or type of stent or UAS, grade of ureteral perforation, degree of bleeding, nor operative time. Furthermore, the registry inherently lacks the granularity to collect subjective metrics such as the amount of resistance while passing the UAS or tightness of the ureter. As such, there are factors unaccounted for in our model. Additionally, our results are based on practices within a single state and may not be generalizable to a national sample. That said, our variation in practice size, geographic location, and academic or private affiliation tempers this potential limitation. Unplanned healthcare utilization is only one of the many objective metrics through which we can measure success following URS and future research should include additional clinical outcomes such as stone-free rate and need for secondary URS however this cannot be addressed in this study.

Limitations notwithstanding our findings are both provocative and novel. We do not capture the cost of these unplanned episodes of care, but they have been previously quantified and amount to a significant financial burden. ${ }^{2}$ Extrapolating from previous data $(\$ 23,436$ per episode $)^{2}$, unplanned healthcare utilization in stented patients during our study period resulted in a $\$ 14,155,344$ increase in cost of care, not accounting for the indirect costs. We found that stent placement increased the odds of an ED visit by $25 \%$, which, in the context of the scope and burden of stone disease, amounts to a substantial clinical significance.

We acknowledge that the decision to place a stent after URS is complex, the significant variation in stent use underscores the uncertainty regarding utilization. There are clearly cases where stent omission is ill-advised. Better defining cases where stent omission is acceptable is of great importance. One of the greatest strengths of a robust continuous QI program like MUSIC ROCKS is that we can actively translate such findings into action. When to place a stent and for how long it should remain in place has been a concern of our member urologists since the early days of MUSIC. To this end, we recently concluded an appropriateness panel to understand clinical scenarios where stent omission can be considered after URS using the RAND-UCLA Methodology. ${ }^{14}$ In addition, ongoing efforts to measure patient reported outcomes following URS in MUSIC should help us better understand the implications of stent omission on quality of life.

\section{Conclusion:}

Ureteral stent placement is commonly performed following URS in Michigan and there exists wide variation in stent use both at a provider- and practice-level, irrespective of case volume. Several factors significantly impact the decision to place a stent. In this analysis, we found stent placement is associated with an increased odds of an ED visit following URS and stent omission appears safe however randomized trials are needed to substantiate these conclusions. These findings have broad implications for patients, in whom stents negatively impact quality of life, as well as the healthcare system. Efforts are underway to operationalize a pragmatic approach to stent use after URS in Michigan as well as continue to evaluate their downstream consequences via patient reported outcomes. 


\section{Acknowledgements:}

MUSIC is funded by Blue Cross and Blue Shield of Michigan (BCBSM) as part of the BCBSM Value Partnerships program. The authors acknowledge the significant contributions of the clinic champions, urologists, administrators, and data abstractors in each participating Michigan Urological Surgery Improvement Collaborative (MUSIC) practice (details around specific participating urologists and practices can be found at www.musicurology.com), as well as members of the MUSIC Coordinating Center at the University of Michigan. In addition, we would like to acknowledge the support provided by the Value Partnerships program at BCBSM.

\section{References}

1. Raheem OA, Khandwala YS, Sur RL, et al. , Burden of Urolithiasis: Trends in Prevalence, Treatments, and Costs. Eur Urol Focus, 2017. 3(1): p. 18-26. [PubMed: 28720363]

2. Scales CD Jr., Saigal CS, Hanley JM, et al. , The impact of unplanned postprocedure visits in the management of patients with urinary stones. Surgery, 2014. 155(5): p. 769-75. [PubMed: 24787103]

3. Mittakanti HR, Conti SL, Pao AC, et al. , Unplanned Emergency Department Visits and Hospital Admissions Following Ureteroscopy: Do Ureteral Stents Make a Difference? Urology, 2018. 117: p. 44-49. [PubMed: 29601836]

4. Joshi HB, Stainthorpe A, MacDonagh RP, et al. , Indwelling ureteral stents: evaluation of symptoms, quality of life and utility. J Urol, 2003. 169(3): p. 1065-9; discussion 1069. [PubMed: 12576847]

5. Assimos D, Krambeck A, Miller NL, et al. , Surgical Management of Stones: American Urological Association/Endourological Society Guideline, PART I. J Urol, 2016. 196(4): p. 115360. [PubMed: 27238616]

6. Türk C, Petřík A, Sarica K, et al. , EAU Guidelines on Interventional Treatment for Urolithiasis. Eur Urol, 2016. 69(3): p. 475-82. [PubMed: 26344917]

7. Muslumanoglu AY, Fuglsig S, Frattini A, et al. , Risks and Benefits of Postoperative Double-J Stent Placement After Ureteroscopy: Results from the Clinical Research Office of Endourological Society Ureteroscopy Global Study. J Endourol, 2017. 31(5): p. 446-451. [PubMed: 28292209]

8. Ordonez M, Hwang EC, Borofsky M, et al. , Ureteral stent versus no ureteral stent for ureteroscopy in the management of renal and ureteral calculi. Cochrane Database Syst Rev, 2019. 2: p. Cd012703. [PubMed: 30726554]

9. Dauw CA, Swarna K, Qi J, et al., Shockwave Lithotripsy Use in the State of Michigan: American Urological Association Guideline Adherence and Clinical Implications. Urology, 2020. 137: p. 3844. [PubMed: 31843621]

10. Dauw CA, Ghani KR, Qi J, et al. , Variable Use of Postoperative Imaging Following Ureteroscopy: Results from a Statewide Quality Improvement Collaborative. Urology, 2020. 136: p. 63-69. [PubMed: 31618657]

11. Charlson ME, Pompei P, Ales KL, et al. , A new method of classifying prognostic comorbidity in longitudinal studies: development and validation. J Chronic Dis, 1987. 40(5): p. 373-83. [PubMed: 3558716]

12. Dauw CA, Simeon L, Alruwaily AF, et al. , Contemporary Practice Patterns of Flexible Ureteroscopy for Treating Renal Stones: Results of a Worldwide Survey. J Endourol, 2015. 29(11): p. 1221-30. [PubMed: 26154856]

13. Pereira JF, Bower P, Jung E, et al. , Ureteral stenting practices following routine ureteroscopy: an international survey. World J Urol, 2019. 37(11): p. 2501-2508. [PubMed: 30747279]

14. Fitch K, Bernstein S, Aguilar M, et al., The RAND/UCLA appropriateness method user's manual 2001, Santa Monica, CA: RAND. 


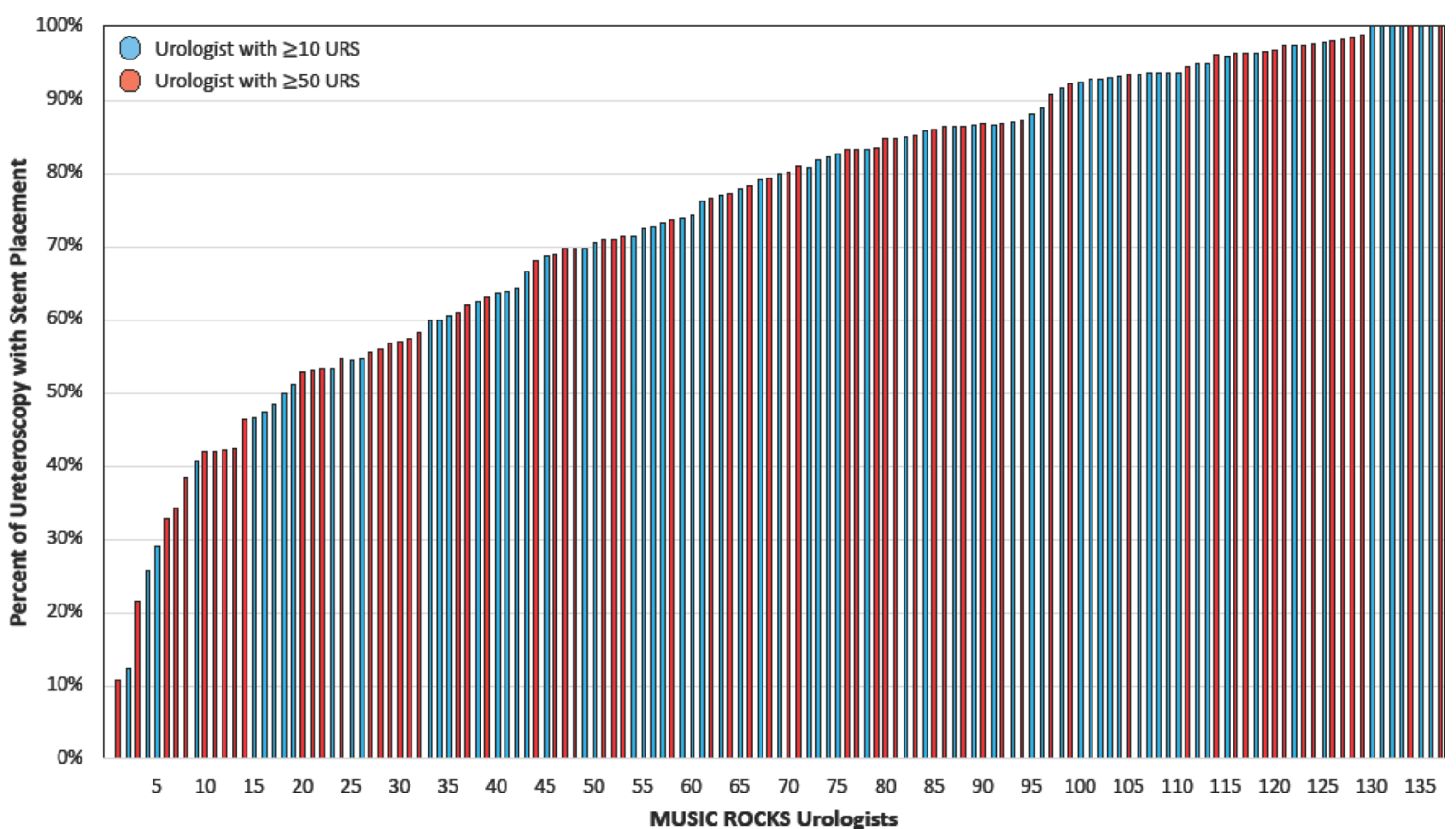

Figure 1.

Variation in rates of ureteral stent placement across urologists in MUSIC ROCKS. 


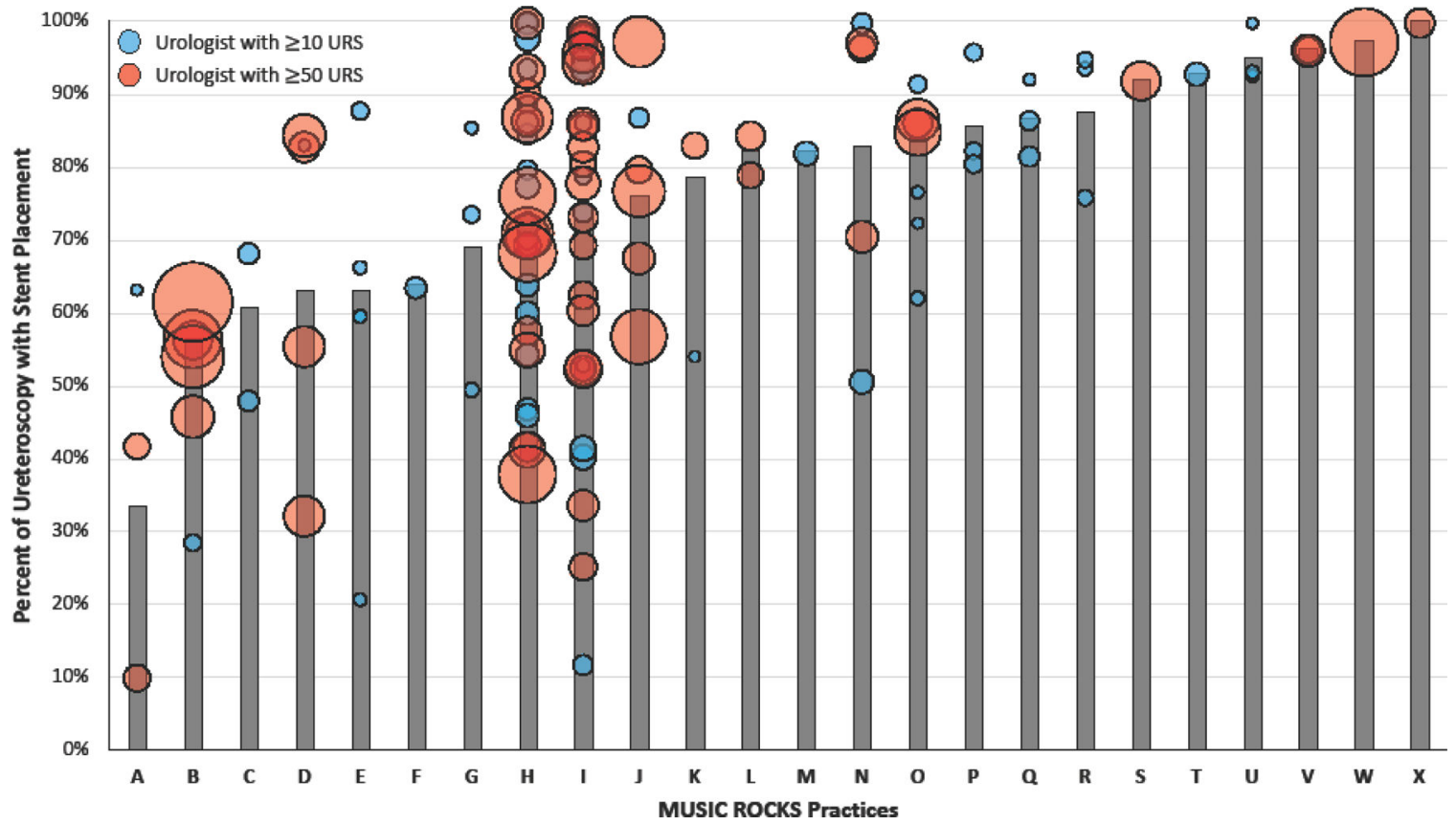

Figure 2.

Variation in rates of stent placement by practice (bars) and by urologist (bubble) within each practice. Size of bubble is scaled to represent total URS case volume for individual urologist. 


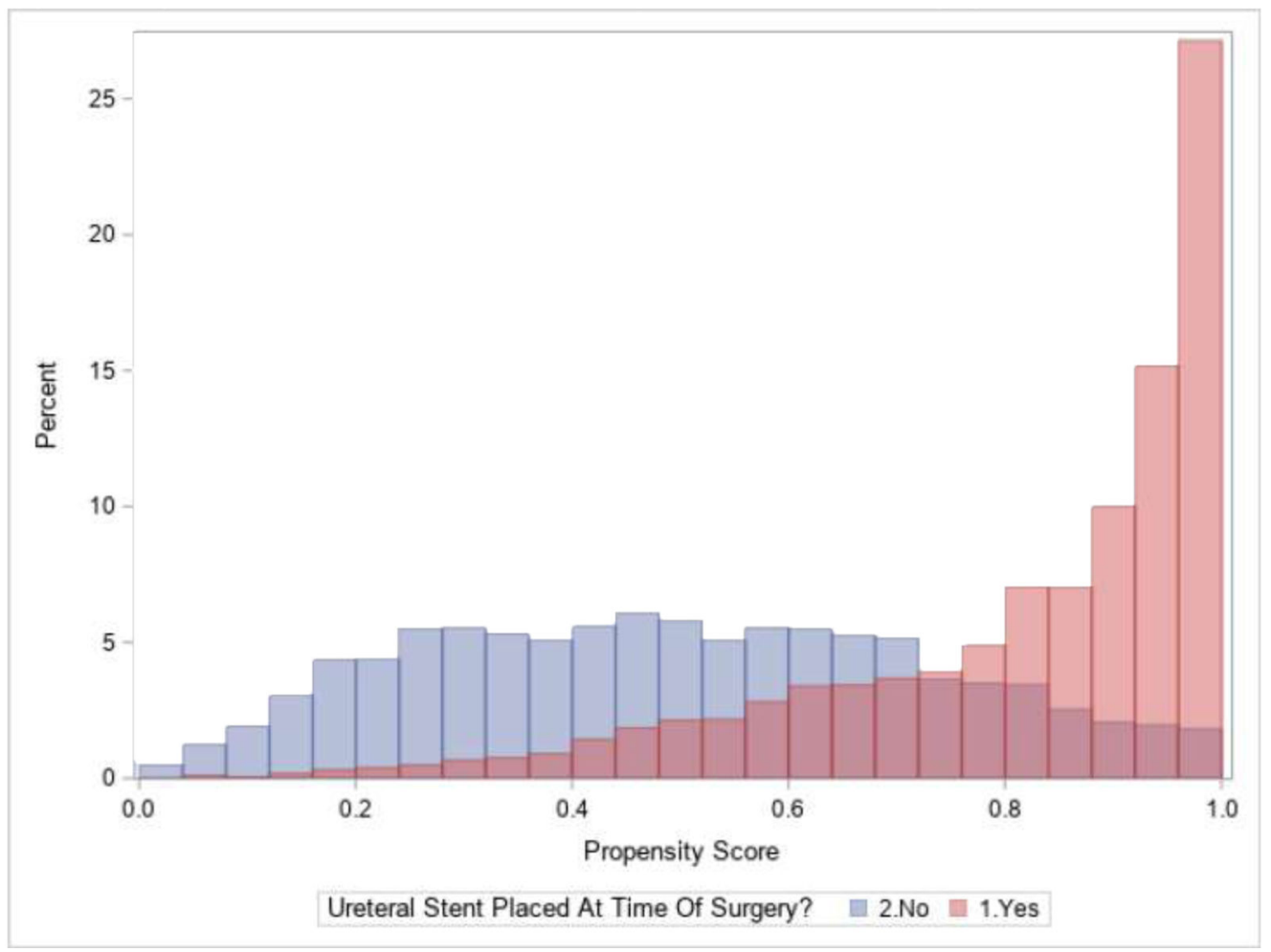

Figure 3.

Propensity scores by those with and without ureteral stent placed at the time of surgery. 
Table 1.

Demographics, clinical characteristics and outcomes in cases with and without stent placement

\begin{tabular}{|c|c|c|c|}
\hline & No Stent & Stent & P-Value \\
\hline URS cases & $2637(27.3 \%)$ & $7025(72.7 \%)$ & \\
\hline Age, mean (SD), years & $53.2(16.4)$ & $55.8(15.9)$ & $<0.001$ \\
\hline \multicolumn{4}{|l|}{ Gender } \\
\hline Male & $1218(46.2 \%)$ & $3514(50.0 \%)$ & $<0.001$ \\
\hline Female & $1419(53.8 \%)$ & $3510(50.0 \%)$ & \\
\hline \multicolumn{4}{|l|}{ Insurance } \\
\hline Private & $1580(60.3 \%)$ & $4050(57.9 \%)$ & 0.004 \\
\hline Public & $972(37.1 \%)$ & $2809(40.2 \%)$ & \\
\hline None & $69(2.6 \%)$ & $135(1.9 \%)$ & \\
\hline \multicolumn{4}{|l|}{ Body mass index } \\
\hline$\underline{25}$ & $517(22.0 \%)$ & $1416(21.6 \%)$ & 0.5 \\
\hline$>25-\unlhd 30$ & $758(32.2 \%)$ & $2057(31.4 \%)$ & \\
\hline$>30-\leq 35$ & $531(22.6 \%)$ & $1513(23.1 \%)$ & \\
\hline$>35$ & $548(23.3 \%)$ & $1569(23.9 \%)$ & \\
\hline \multicolumn{4}{|l|}{ Charlson Comorbidity Index } \\
\hline 0 & $1898(72.0 \%)$ & $4855(69.1 \%)$ & 0.2 \\
\hline 1 & $358(13.6 \%)$ & $1200(17.1 \%)$ & \\
\hline 22 & $379(14.4 \%)$ & $967(13.8 \%)$ & \\
\hline \multicolumn{4}{|l|}{ Preoperative urine culture result } \\
\hline Positive & $319(12.1 \%)$ & $862(12.3 \%)$ & 0.6 \\
\hline Negative & $1775(67.4 \%)$ & $4779(68.1 \%)$ & \\
\hline Not performed & $541(20.5 \%)$ & $1376(19.6 \%)$ & \\
\hline Pre-stented & $1337(50.8 \%)$ & $2443(35.1 \%)$ & $<0.001$ \\
\hline Stone size, mean (SD), millimeters & $6.1(2.8)$ & $7.6(3.5)$ & $<0.001$ \\
\hline \multicolumn{4}{|l|}{ Stone size, diameter } \\
\hline $5 \mathrm{~mm}$ & $1202(47.7 \%)$ & $1987(29.6 \%)$ & $<0.001$ \\
\hline$>5 \mathrm{~mm}-\unlhd 10 \mathrm{~mm}$ & $1144(45.4 \%)$ & $3527(52.5 \%)$ & \\
\hline$>10 \mathrm{~mm}$ & $176(7.0 \%)$ & $1209(18.0 \%)$ & \\
\hline \multicolumn{4}{|l|}{ Stone location } \\
\hline Ureter & $1542(63.5 \%)$ & $3858(59.4 \%)$ & 0.002 \\
\hline Kidney & $528(21.8 \%)$ & $1522(24.1 \%)$ & \\
\hline Both & $358(14.7 \%)$ & $1068(16.5 \%)$ & \\
\hline Ureteral access sheath use & $395(15.1 \%)$ & $3096(44.9 \%)$ & $<0.001$ \\
\hline Intraoperative complication & $9(0.3 \%)$ & $73(1.0 \%)$ & $<0.001$ \\
\hline Bleeding & $8(88.9 \%)$ & $50(68.5 \%)$ & \\
\hline Perforation & $1(11.1 \%)$ & $23(31.5 \%)$ & \\
\hline Emergency department visit & $186(7.1 \%)$ & $599(8.5 \%)$ & 0.018 \\
\hline
\end{tabular}




\begin{tabular}{|l|c|c|c|}
\hline & No Stent & Stent & P-Value \\
\hline Hospitalization & $76(2.9 \%)$ & $267(3.8 \%)$ & 0.030 \\
\hline
\end{tabular}


Table 2.

Multivariable model assessing risk factors for stent placement at the time of ureteroscopy adjusting for provider and practice variation.

\begin{tabular}{|c|c|c|c|}
\hline & Adjusted Odds Ratio & 95\% Confidence Interval & P-Value \\
\hline Age (unit change from mean) & 1.01 & $1.00-1.01$ & $<0.001$ \\
\hline Gender & & & 0.079 \\
\hline Male (vs. female) & 1.12 & $0.99-1.27$ & \\
\hline BMI & & & 0.6 \\
\hline$\underline{25}$ (vs. >35) & 1.01 & $0.84-1.22$ & \\
\hline$>25-\unlhd 30($ vs. $>35)$ & 0.91 & $0.77-1.07$ & \\
\hline$>30-\$ 35($ vs. $>35)$ & 0.97 & $0.81-1.17$ & \\
\hline Insurance & & & 0.4 \\
\hline Private (vs. public) & 0.97 & $0.85-1.12$ & \\
\hline None (vs. public) & 0.76 & $0.49-1.17$ & \\
\hline Charlson Comorbidity Index & & & 0.4 \\
\hline 1 (vs. 0) & 1.13 & $0.94-1.35$ & \\
\hline$\geq 2($ vs. 0$)$ & 1.00 & $0.83-1.21$ & \\
\hline Urine culture & & & 0.5 \\
\hline Positive (vs. negative) & 1.11 & $0.91-1.35$ & \\
\hline Not performed (vs. negative) & 0.97 & $0.81-1.16$ & \\
\hline Pre-stented (vs. no) & 0.25 & $0.22-0.29$ & $<0.001$ \\
\hline Stone size, diameter & & & $<0.001$ \\
\hline$>5 \mathrm{~mm}-\unlhd 10 \mathrm{~mm}$ (vs. $5 \mathrm{~mm})$ & 1.89 & $1.65-2.15$ & \\
\hline$>10 \mathrm{~mm}(\mathrm{vs} .5 \mathrm{~mm})$ & 4.68 & $3.70-5.92$ & \\
\hline Stone location & & & $<0.001$ \\
\hline Kidney (vs. ureter) & 0.69 & $0.59-0.82$ & \\
\hline Both (vs. ureter) & 0.99 & $0.83-1.19$ & \\
\hline Ureteral access sheath (vs. no) & 5.43 & $4.57-6.46$ & $<0.001$ \\
\hline Intraoperative complication (vs. no) & 2.18 & $0.92-5.20$ & 0.078 \\
\hline
\end{tabular}


Table 3.

Reason for ED visit in patients with or without a ureteral stent.

\begin{tabular}{|l|c|c|c|}
\hline & No Stent, $\mathbf{N}(\boldsymbol{\%})$ & Stent, $\mathbf{N}(\boldsymbol{\%})$ & Total \\
\hline ED visit (N=) & 186 & 599 & 785 \\
\hline Flank pain & $109(58.6)$ & $317(52.9)$ & $426(54.3)$ \\
\hline Other & $69(37.1)$ & $212(35.4)$ & $281(35.8)$ \\
\hline Hematuria & $29(15.6)$ & $113(18.9)$ & $142(18.1)$ \\
\hline Urinary tract infection & $26(14.0)$ & $113(18.9)$ & $139(17.7)$ \\
\hline Fever & $26(14.0)$ & $79(13.2)$ & $105(13.4)$ \\
\hline Nausea & $20(10.8)$ & $76(12.7)$ & $96(12.2)$ \\
\hline Abdominal pain & $10(5.4)$ & $41(6.8)$ & $51(6.5)$ \\
\hline Dysuria & $8(4.3)$ & $38(6.3)$ & $46(5.9)$ \\
\hline Sepsis & $13(7.0)$ & $32(5.3)$ & $45(5.7)$ \\
\hline Urinary frequency & $7(3.8)$ & $30(5.0)$ & $37(4.7)$ \\
\hline Urinary retention & $10(5.4)$ & $24(4.0)$ & $34(4.3)$ \\
\hline Stent displacement & $0(0)$ & $17(2.8)$ & $17(2.2)$ \\
\hline Bladder pain & $2(1.1)$ & $11(1.8)$ & $13(1.7)$ \\
\hline Syncope & $0(0)$ & $9(1.5)$ & $9(1.1)$ \\
\hline Obstructing stone & $2(1.1)$ & $5(0.8)$ & $7(0.9)$ \\
\hline Deep venous thrombosis & $0(0)$ & $4(0.7)$ & $4(0.5)$ \\
\hline Renal failure & $1(0.5)$ & $3(0.5)$ & $4(0.5)$ \\
\hline Peri-renal hematoma & $0(0)$ & $2(0.3)$ & $2(0.3)$ \\
\hline Ureteral injury & $1(0.5)$ & $0(0)$ & $1(0.1)$ \\
\hline Cerebrovascular accident & $0(0)$ & $1(0.2)$ & $1(0.1)$ \\
\hline
\end{tabular}


Table 4.

Multivariable analysis assessing odds of ED visit and hospitalization following ureteroscopy associated with ureteral stent placement adjusting for provider and practice variation.

\begin{tabular}{|c|c|c|c|c|c|c|}
\hline & \multicolumn{3}{|c|}{$\underline{\text { ED Visit }}$} & \multicolumn{3}{|c|}{$\underline{\text { Hospitalization }}$} \\
\hline & $\begin{array}{c}\text { Adjusted } \\
\text { Odds Ratio }\end{array}$ & $\begin{array}{l}\text { 95\% Confidence } \\
\text { Interval }\end{array}$ & P-Value & $\begin{array}{c}\text { Adjusted } \\
\text { Odds Ratio }\end{array}$ & $\begin{array}{l}\text { 95\% Confidence } \\
\text { Interval }\end{array}$ & P-Value \\
\hline Stent placement (vs. no) & 1.25 & $1.01-1.54$ & 0.0428 & 1.28 & $0.94-1.76$ & 0.12 \\
\hline $\begin{array}{l}\text { Age (unit change from } \\
\text { mean) }\end{array}$ & 0.99 & $0.98-0.99$ & $<0.001$ & 0.99 & $0.98-1.00$ & 0.012 \\
\hline Gender & & & 0.011 & & & 0.6 \\
\hline Male (vs. female) & 0.87 & $0.74-1.03$ & & 1.06 & $0.83-1.35$ & \\
\hline BMI & & & 0.7 & & & 0.9 \\
\hline$\underline{25}$ (vs. >35) & 0.89 & $0.70-1.14$ & & 1.10 & $0.78-1.56$ & \\
\hline$>25-\leq 30$ (vs. $>35)$ & 0.95 & $0.76-1.18$ & & 1.04 & $0.75-1.44$ & \\
\hline$>30-\leq 35$ (vs. $>35)$ & 1.02 & $0.81-1.28$ & & 0.96 & $0.67-1.37$ & \\
\hline Insurance & & & 0.3 & & & 0.03 \\
\hline Private (vs. public) & 0.87 & $0.73-1.04$ & & 0.73 & $0.56-0.94$ & \\
\hline None (vs. public) & 0.84 & $0.46-1.53$ & & 0.43 & $0.13-1.38$ & \\
\hline $\begin{array}{l}\text { Charlson Comorbidity } \\
\text { Index }\end{array}$ & & & $<0.001$ & & & $<0.001$ \\
\hline 1 (vs. 0 ) & 1.26 & $1.00-1.58$ & & 1.57 & $1.14-2.17$ & \\
\hline$\geq 2$ (vs. 0 ) & 1.59 & $1.26-2.02$ & & 2.19 & $1.59-3.02$ & \\
\hline Urine culture & & & 0.007 & & & 0.005 \\
\hline Positive (vs. negative) & 1.27 & $1.00-1.61$ & & 1.54 & $1.12-2.13$ & \\
\hline $\begin{array}{r}\text { Not performed (vs. } \\
\text { negative) }\end{array}$ & 0.77 & $0.60-0.98$ & & 0.79 & $0.55-1.13$ & \\
\hline Pre-stented (vs. no) & 0.73 & $0.60-0.88$ & 0.001 & 1.02 & $0.78-1.33$ & 0.9 \\
\hline Stone size, diameter & & & $<0.001$ & & & $<0.001$ \\
\hline$>5 \mathrm{~mm}-\unlhd 10 \mathrm{~mm}(\mathrm{vs}$. & 0.69 & $0.57-0.83$ & & 0.56 & $0.42-0.74$ & \\
\hline$>10 \mathrm{~mm}$ (vs. $5 \mathrm{~mm})$ & 0.81 & $0.63-1.05$ & & 0.9 & $0.63-1.28$ & \\
\hline Stone location & & & $<0.001$ & & & 0.029 \\
\hline Kidney (vs. ureter) & 1.39 & $1.13-1.70$ & & 1.42 & $1.06-1.91$ & \\
\hline Both (vs. ureter) & 1.47 & $1.18-1.83$ & & 1.41 & $1.02-1.95$ & \\
\hline $\begin{array}{l}\text { Ureteral access sheath (vs. } \\
\text { no) }\end{array}$ & 1.22 & $1.01-1.48$ & 0.043 & 1.25 & $0.95-1.64$ & 0.12 \\
\hline $\begin{array}{l}\text { Intraoperative complication } \\
\text { (vs. no) }\end{array}$ & 2.40 & $1.27-4.55$ & 0.007 & 1.5 & $0.53-4.25$ & 0.4 \\
\hline
\end{tabular}




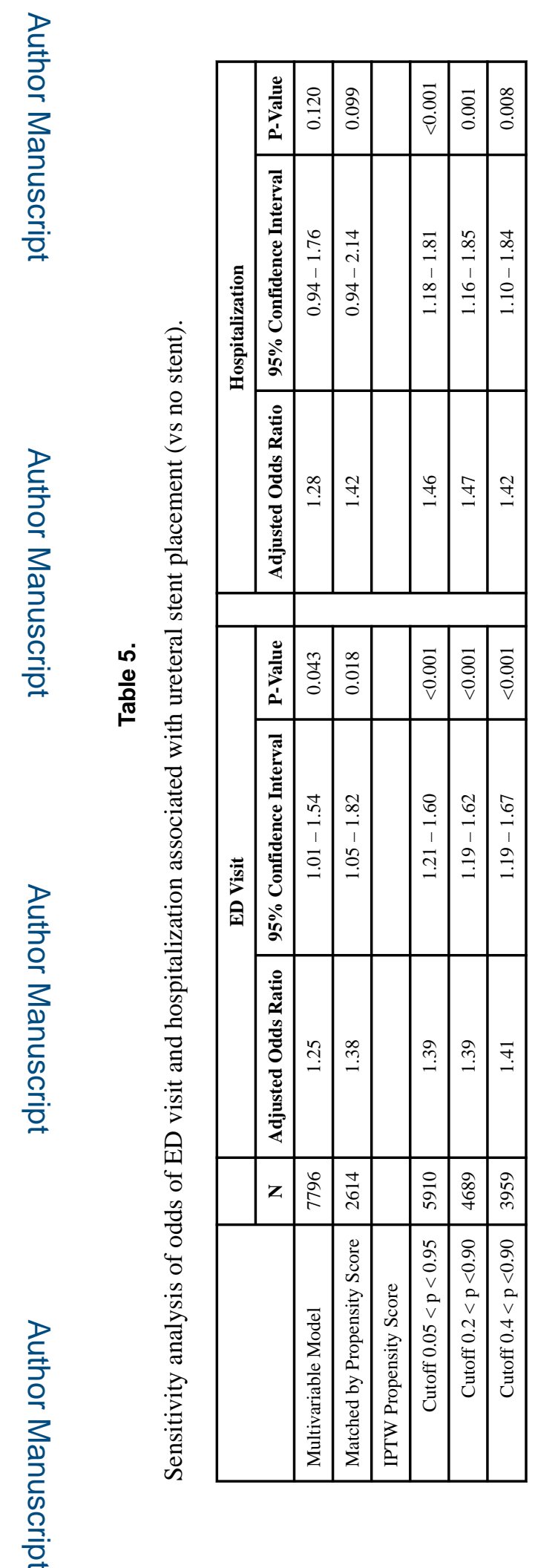

J Urol. Author manuscript; available in PMC 2021 October 11. 